\title{
The evolution of luminous red nova AT 2017jfs in NGC 4470
}

\author{
A. Pastorello ${ }^{1}$, T.-W. Chen ${ }^{2}$, Y.-Z. Cai ${ }^{1,3}$, A. Morales-Garoffolo ${ }^{4}$, Z. Cano ${ }^{5}$, E. Mason ${ }^{6}$, E. A. Barsukova ${ }^{7}$, \\ S. Benetti ${ }^{1}$, M. Berton ${ }^{8,9}$, S. Bose ${ }^{10}$, F. Bufano ${ }^{11}$, E. Callis ${ }^{12}$, G. Cannizzaro ${ }^{13,14}$, R. Cartier ${ }^{15}$, Ping Chen ${ }^{10}$, \\ Subo Dong ${ }^{10}$, S. Dyrbye ${ }^{16}$, N. Elias-Rosa ${ }^{17,18}$, A. Flörs ${ }^{19,20,21}$, M. Fraser ${ }^{12}$, S. Geier ${ }^{22,23}$, V. P. Goranskij ${ }^{24}$,
} D. A. Kann ${ }^{5}$, H. Kuncarayakti ${ }^{8,25}$, F. Onori ${ }^{26}$, A. Reguitti ${ }^{27}$, T. Reynolds ${ }^{25}$, I. R. Losada ${ }^{28,29}$, A. Sagués Carracedo ${ }^{30}$, T. Schweyer ${ }^{2}$, S. J. Smartt ${ }^{31}$, A. M. Tatarnikov ${ }^{24}$, A. F. Valeev ${ }^{7,32}$, C. $\operatorname{Vogl}^{20,21}$, T. Wevers ${ }^{33}$, A. de Ugarte Postigo ${ }^{5,34}$, L. Izzo $^{5}$, C. Inserra ${ }^{35}$, E. Kankare ${ }^{25}$, K. Maguire ${ }^{31,36}$, K. W. Smith ${ }^{31}$, B. Stalder ${ }^{37}$, L. Tartaglia ${ }^{38}$, C. C. Thöne ${ }^{5}$, G. Valerin ${ }^{3}$, and D. R. Young ${ }^{31}$

(Affiliations can be found after the references)

Received 21 March 2019 / Accepted 23 April 2019

\begin{abstract}
We present the results of our photometric and spectroscopic follow-up of the intermediate-luminosity optical transient AT 2017jfs. At peak, the object reaches an absolute magnitude of $M_{g}=-15.46 \pm 0.15 \mathrm{mag}$ and a bolometric luminosity of $5.5 \times 10^{41} \mathrm{erg} \mathrm{s}^{-1}$. Its light curve has the doublepeak shape typical of luminous red novae (LRNe), with a narrow first peak bright in the blue bands, while the second peak is longer-lasting and more luminous in the red and near-infrared (NIR) bands. During the first peak, the spectrum shows a blue continuum with narrow emission lines of $\mathrm{H}$ and Fe II. During the second peak, the spectrum becomes cooler, resembling that of a K-type star, and the emission lines are replaced by a forest of narrow lines in absorption. About 5 months later, while the optical light curves are characterized by a fast linear decline, the NIR ones show a moderate rebrightening, observed until the transient disappears in solar conjunction. At these late epochs, the spectrum becomes reminiscent of that of M-type stars, with prominent molecular absorption bands. The late-time properties suggest the formation of some dust in the expanding common envelope or an IR echo from foreground pre-existing dust. We propose that the object is a common-envelope transient, possibly the outcome of a merging event in a massive binary, similar to NGC 4490-2011OT1.
\end{abstract}

Key words. binaries: close - stars: massive - supernovae: individual: AT 2017jfs - supernovae: individual: NGC 440-2011OT1 stars: winds, outflows

\section{Introduction}

Red Novae (RNe) form a family of optical transients spanning an enormous range of luminosities. This includes faint objects with absolute peak magnitudes $M_{V}$ from -4 to -6.5 mag, such as OGLE 2002-BLG-360 (Tylenda et al. 2013) and V1309 Sco (Mason et al. 2010; Tylenda et al. 2011), intermediate-luminosity events $\left(M_{V} \gtrsim-10 \mathrm{mag}\right)$ like V838 Mon (Munari et al. 2002; Goranskij et al. 2002; Kimeswenger et al. 2002; Crause et al. 2003), and relatively luminous objects such as NGC 4490-20110T1 (Smith et al. 2016), that can reach $M_{V} \approx-15$ mag. Objects brighter than $M_{V}=-10$ mag are conventionally named luminous red novae ${ }^{1}$ (LRNe; for a review, see Pastorello et al. 2019, and references therein).

Although the physical processes triggering these outbursts have been debated, there is growing evidence that $\mathrm{RNe}$ and their more luminous counterparts are produced by the coalescence of stars with different masses following a commonenvelope phase (e.g., Kochanek et al. 2014; Pejcha et al. 2016, 2017; MacLeod et al. 2017, 2018). In particular, the inspiralling motion of the secondary was revealed by the long-term monitoring of V1309 Sco (Tylenda et al. 2011).

\footnotetext{
* Table A.1 is only available at the CDS via anonymous ftp to cdsarc.u-strasbg.fr (130.79.128.5) or via http://cdsarc. u-strasbg.fr/viz-bin/qcat?]/A+A/625/L8

1 The alternative naming "luminous red variable" was also used in the past (e.g., Martini et al. 1999).
}

The recent discovery of LRNe suggests that common envelope ejections and/or merging events may also happen in more massive close binary systems (Smith et al. 2016; Mauerhan et al. 2018), with major implications for the evolution of the resulting merger. In this context, here we report the results of our follow-up campaign of a LRN recently discovered in the galaxy NGC 4470: AT 2017jfs.

\section{AT 2017jfs, its host galaxy, and reddening}

AT 2017jfs ${ }^{2}$ was discovered by Gaia on 2017 December 26.13 UT (MJD = 58113.13, Delgado 2007) at a Gaia G-band magnitude $17.17 \pm 0.20$. No source was detected at the transient position on 2017 November 30 down to a limiting magnitude of 21.5. The transient was located at $\alpha=12^{\mathrm{h}} 29^{\mathrm{m}} 37 \mathrm{~s} .79$ and $\delta=+07^{\circ} 49^{\prime} 35^{\prime \prime} .18$ (equinox J2000.0), in the almost face-on, early-type spiral galaxy NGC 4470.

The source was tentatively classified by the extended-Public ESO Spectroscopic Survey for Transient Objects (ePESSTO; Smartt et al. 2015) as a type-IIn supernova (SN IIn) or a SN impostor (Bufano et al. 2018), and for this reason it was designated with a SN name (SN 2017jfs). In this paper, we show it to be a LRN, hence we adopt the label AT 2017jfs.

2 The object is known by multiple survey designations, including Gaia17dkh, PS17fqp, ATLAS18aat. 


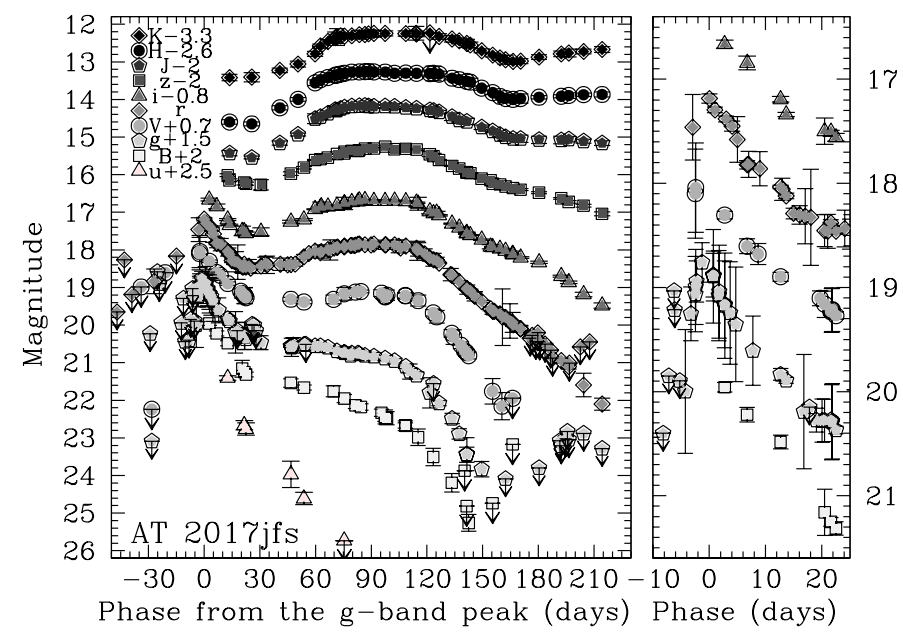

Fig. 1. Left: optical and NIR light curves of AT 2017jfs. The phases are calculated from the first $g$-band peak, on MJD $=58114.8 \pm 1.8$. Only the most significant detection limits are shown. Right: $B, V, g, r$ and $i$ light curves during the first (blue) peak.

The distance to NGC 4470 is somewhat controversial, and the NASA/IPAC Extragalactic Database (NED) ${ }^{3}$ gives a number of discrepant estimates based on the Tully-Fisher method, ranging from 11.6 to $34.6 \mathrm{Mpc}$, with an average value of $18.76 \pm 6.6 \mathrm{Mpc}$ (corresponding to a distance modulus $\mu=$ $31.25 \pm 0.70 \mathrm{mag}$ ). Given the uncertainty in the Tully-Fisher estimates, we prefer to adopt a kinematic distance $d=$ $35.2 \pm 2.7 \mathrm{Mpc}$ (corrected for Virgo Infall and estimated adopting a standard cosmology with $H_{0}=73 \mathrm{~km} \mathrm{~s}^{-1} \mathrm{Mpc}^{-1}$ ), hence $\mu=32.73 \pm 0.15 \mathrm{mag}$. This estimate also agrees with the distance $d \sim 34.7 \mathrm{Mpc}$ reported by Koliopanos et al. (2017).

The Galactic line-of-sight reddening is modest, $E(B-V)=$ $0.022 \mathrm{mag}$ (Schlafly \& Finkbeiner 2011). Our early spectra do not have high signal to noise ratios ( $\mathrm{S} / \mathrm{Ns}$ ), and therefore the host galaxy reddening cannot be well constrained. However, prominent absorption features of Na ID are not visible, suggesting a modest host galaxy reddening contribution. Later spectra with higher S/N show only a narrow Na ID in absorption centered at $5884 \AA$ (rest wavelength), hence likely a feature intrinsic to AT 2017 jfs. For this reason, we adopt $E(B-V)=0.022$ mag as the total reddening towards AT 2017jfs.

\section{Photometric evolution}

The follow-up campaign started soon after the classification of AT 2017jfs, and continued for about 7 months. Photometry data were reduced following standard prescriptions (see, e.g., Cai et al. 2018), using the SNOoPy package (Cappellaro 2014) The magnitudes are listed in Table A.1, available at the CDS, which contains the following information: Column 1 lists the date of the observation, Col. 2 lists the MJD, Cols. 3-11 give the optical and near-infrared (NIR) magnitudes, and Col. 12 reports a numeric code for the instrumental configuration. The multi-band light curves are shown in Fig. 1. The Sloan- $u$ light curve shows a monotonic decline after maximum, with an average rate of $6.5 \pm 0.9 \mathrm{mag}(100 \mathrm{~d})^{-1}$. The photometric evolution in the other bands is somewhat different. The $g$-band maximum is constrained to MJD $=58114.8 \pm 1.8(2017$ December $27.8 \mathrm{UT})$ through a low-order polynomial fit (at $g=17.35 \pm 0.02 \mathrm{mag}$, hence $M_{g}=-15.46 \pm 0.15 \mathrm{mag}$ ).

\footnotetext{
https://ned.ipac.caltech.edu/
}

The $g$-band light curve has a rise time to maximum of about $4 \mathrm{~d}$, followed by a rapid decline $\left(6.5 \pm 0.2 \mathrm{mag}(100 \mathrm{~d})^{-1}\right)$ until $\sim 50 \mathrm{~d}$. The light curve follows a plateau-like evolution until $\sim 110 \mathrm{~d}$ when it begins a faster decline $\left(7.0 \pm 0.4 \mathrm{mag}(100 \mathrm{~d})^{-1}\right)$ that lasts until it has faded below the detection threshold. The evolution in the Johnson $B$ and $V$ bands is similar to the $g$ band, although the $V$-band light curve shows a low-contrast second peak, broader than the early one.

The light curve is remarkably different in the red and NIR bands. The transient reaches a peak at $r=17.19 \pm 0.05 \mathrm{mag}$ on $\mathrm{MJD}=58115.6$, followed by a fast decline $(6.3 \pm 0.3 \mathrm{mag}$ $\left.(100 \mathrm{~d})^{-1}\right)$ lasting three weeks and reaching a minimum at $r=$ $18.32 \pm 0.07$ mag. Subsequently, from about $50 \mathrm{~d}$ after maximum, the $r$-band luminosity rises again and reaches a second maximum on $\mathrm{MJD}=58209.0$, at $r=17.68 \pm 0.03 \mathrm{mag}$. This second peak is much broader than the early one. Later on, from 110 to $200 \mathrm{~d}$ after the first peak, the $r$-band light curve declines with a rate of $3.90 \pm 0.04 \mathrm{mag}(100 \mathrm{~d})^{-1}$. The $i$-band light curve is very similar, with the two peaks reaching comparable luminosities.

The NIR light curves have a second maximum, brighter than the early one. As an additional feature, GROND (Greiner et al. 2008) observations reveal a moderate rebrightening of the NIR light curves from $\sim 170$ to $220 \mathrm{~d}$. Although we do not have very late spectroscopic observations to support this (Sect. 4), a latetime NIR luminosity excess can be associated with the formation of new dust or IR echoes, occasionally observed in LRNe at late phases (see, e.g., Banerjee et al. 2015; Exter 2016). The late NIR brightening may also be a consequence of the transition to the brown (L-type) supergiant stage, as happened for V838 Mon (Evans et al. 2003; Munari et al. 2007), although this scenario does not comfortably explain the late blue-shift of the $\mathrm{H} \alpha$ emission observed in the late spectra of AT 2017jfs (see Sect. 4).

\section{Spectral evolution}

We collected 14 epochs of optical spectroscopy, spanning about six months of the evolution of AT 2017jfs. Information on the instrumental configurations is given in Table A.2. Our spectral sequence of AT 2017jfs is presented in Fig. 2, while the comparison with a few LRNe at similar epochs and the line identification are shown in Fig. 3. We remark that the transient lies in a crowded region of NGC 4470, rich in nearby sources. As a consequence, the late spectra show some contamination from host galaxy lines.

The spectral evolution of AT 2017jfs follows a three-phase behavior, as observed in other extra-galactic LRNe. In particular, we note a remarkable similarity with NGC 4490-2011OT1 (Smith et al. 2016; Pastorello et al. 2019) at all phases. At early epochs (until 3-4 weeks after the first $g$-band peak) the spectrum of AT 2017jfs shows a blue continuum, dominated by prominent $\mathrm{H}$ lines in emission, with a Lorentzian profile and a full width at half maximum velocity $v_{F W H M} \sim 700 \mathrm{~km} \mathrm{~s}^{-1}$ (corrected for spectral resolution). In this period, the temperature inferred from a black-body fit to the spectral continuum, $T_{\mathrm{BB}}$, decreases from about $7800 \pm 700 \mathrm{~K}$ (in the $+9.5 \mathrm{~d}$ spectrum) to $6000 \pm 600 \mathrm{~K}$ (in the $+23.4 \mathrm{~d}$ spectrum). Emission lines from a number of Fe II multiplets are also detected, along with $\mathrm{O}$ I. The $\mathrm{Ca}$ II NIR triplet is also identified in emission, while the $H \& \mathrm{~K}$ feature, which is usually prominent in absorption in other LRNe (see Fig. 3, top panel), is marginally detected in AT 2017jfs.

With time, the continuum becomes redder and the spectrum experiences an evident metamorphosis. During the second peak, from $\sim 50 \mathrm{~d}$ to 4 months, the red spectrum $\left(T_{\mathrm{bb}}=4300 \pm 700 \mathrm{~K}\right.$ at $82.3 \mathrm{~d}$ ) is dominated by a forest of metal lines in absorption. 


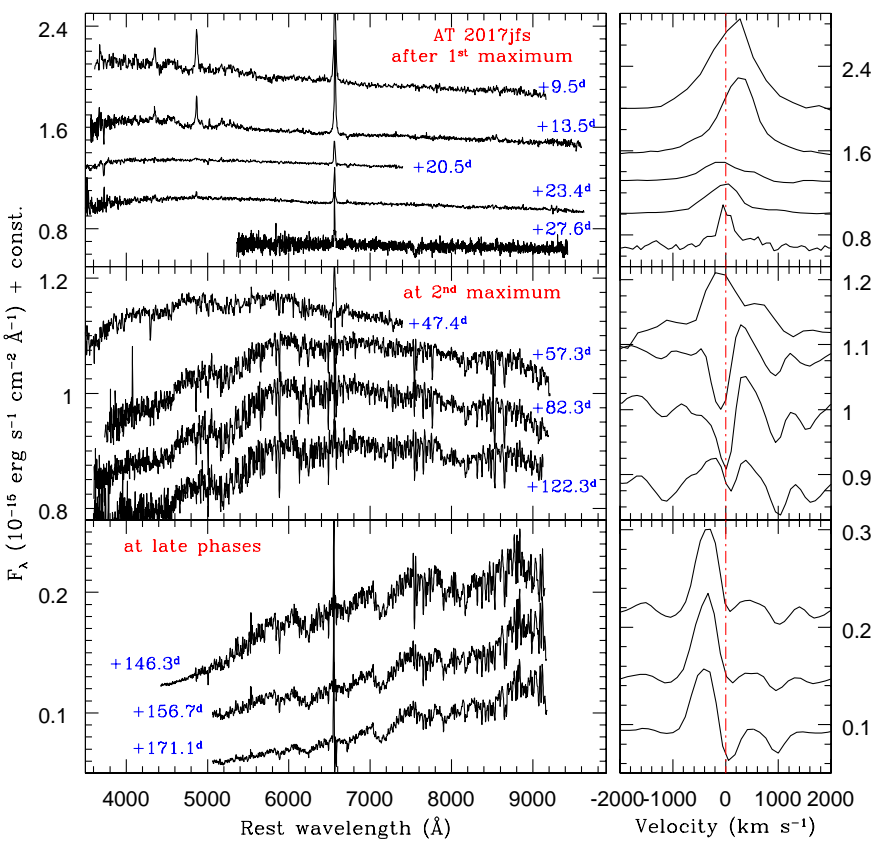

Fig. 2. Left: spectral evolution of AT 2017jfs soon after the first blue maximum (top), around the second, red maximum (center), and at late phases (bottom). Right: expanded image of the $\mathrm{H} \alpha$ region in the velocity space. The vertical line marks the rest wavelength position of $\mathrm{H} \alpha$ at the same phases as the left panels (after correcting for the host galaxy redshift $z=0.007809$ as reported in NED). Only the highest-S/N spectra are shown. The absorptions visible at velocities 0 and $+1000 \mathrm{~km} \mathrm{~s}^{-1}$ in late spectra can be partly due to an over-subtraction of the unresolved $H \alpha$ and [N II] $\lambda 6583$ from the host galaxy background.

The $\mathrm{H}$ lines become much weaker, showing now a $\mathrm{P}$ Cygni profile (see Fig. 2, left panel), although an over-subtraction of the narrow $\mathrm{H} \alpha$ emission from a nearby $\mathrm{H}$ II region may affect the apparent strength of the absorption. The spectrum in this phase is reminiscent of intermediate-type stars (e.g., late $\mathrm{G}$ to $\mathrm{K}$ types). We identify a number of metal lines (from Fe II, Ti II, Sc II and Ba II multiplets), along with the Na I 5889, $5895 \AA$ doublet (see Fig. 3, mid panel). The O I and the NIR Ca II triplet are now seen in absorption. The velocity of the narrow Fe II lines deduced from the wavelengths of absorptions is about $450 \mathrm{~km} \mathrm{~s}^{-1}$. Some of the absorption lines visible at this stage are likely due to neutral metals, in particular Fe I at red wavelengths.

From about 5 months after maximum (hence during the steep, late luminosity decline; see Sect. 3), the spectrum changes again, becoming much redder $\left(T_{\mathrm{bb}} \approx 2950 \pm 150 \mathrm{~K}\right.$ at $\left.157 \mathrm{~d}\right)$ and closer to that of an M-type star. $\mathrm{H} \alpha$ is now mostly in emission, with an evident blue-shift of its peak (by about $400 \mathrm{~km} \mathrm{~s}^{-1}$; see Fig. 2, right), and a deep absorption at the rest velocity. While an over-subtraction of the contaminant HII region can be responsible in part for this absorption, the strong blueshift of the emission is real. The $\mathrm{H} \alpha$ profile is similar to that of LRN NGC 4490-2011OT1 about 200 days after maximum (Smith et al. 2016), whose bluest emission peak was shifted by $-280 \mathrm{~km} \mathrm{~s}^{-1}$. Following Smith et al. (2016), the development of a blue-shifted component in emission would be consistent with an expanding, shock-heated line-forming region, possibly with aspherical symmetry. A peculiar geometry or, alternatively, the formation of dust hiding the rear emitting region (or both) may explain the late $\mathrm{H} \alpha$ profile for both NGC 4490-2011OT1 and AT 2017jfs. Higher-resolution spectra and a wider temporal monitoring would help in discriminating the different scenarios.
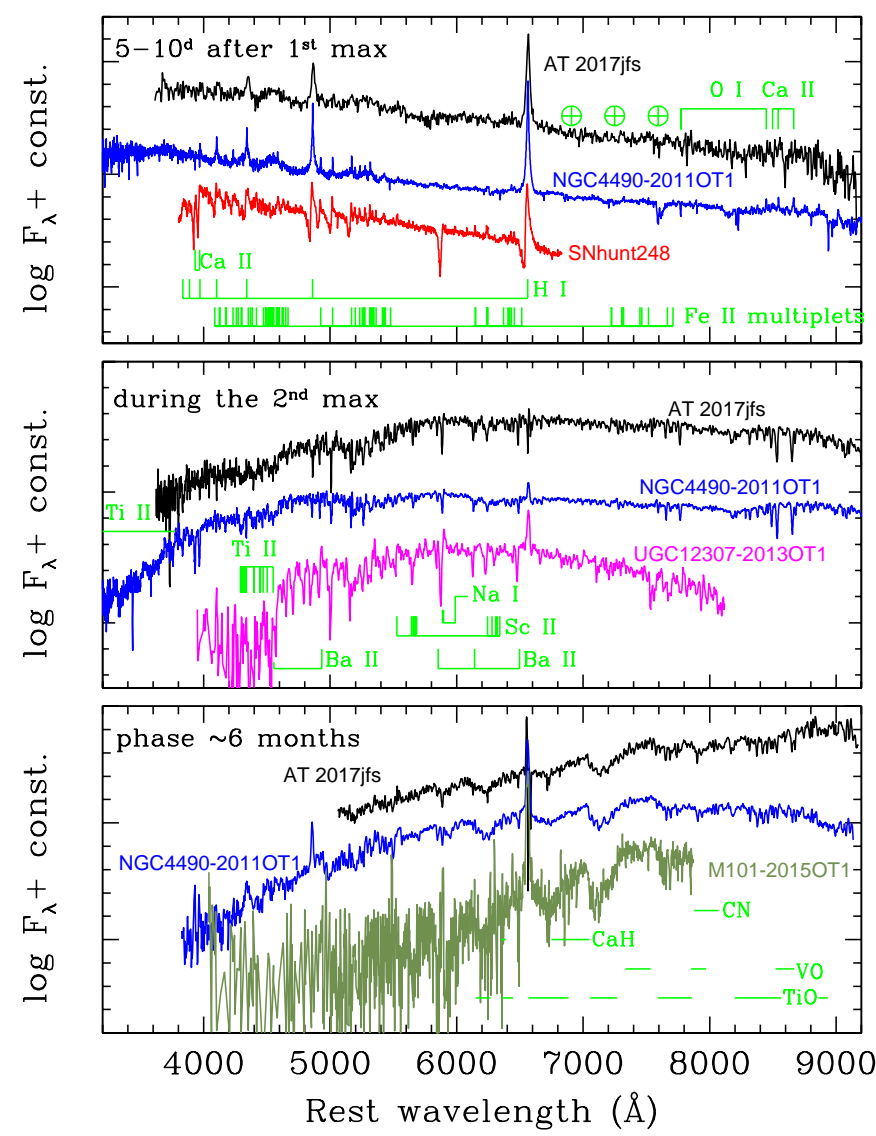

Fig. 3. Line identification in the spectra of AT 2017jfs and a few comparison LRNe at three representative phases: a few days after the blue peak (top panel), around the red peak (middle panel), and at late phases (about 5-6 months after the blue peak; bottom panel). The spectra of the comparison objects are taken from Pastorello et al. (2019), Blagorodnova et al. (2017), and Kankare et al. (2015). The identification of the molecular bands is performed following Kirkpatrick et al. (1991), Valenti et al. (1998), Martini et al. (1999), and Barsukova et al. (2014).

As observed in similar transients (e.g., Pastorello et al. 2019), the late-time spectrum is also characterized by broad absorption bands. The features are generally identified as being due to molecules, in particular $\mathrm{TiO}$ and $\mathrm{VO}$, although $\mathrm{CN}$ and $\mathrm{CaH}$ are not ruled out (see Fig. 3, top panel).

\section{Evolution of the temperature and the radius}

The double-peaked light-curve evolution and the major spectroscopic transition from an SN IIn-like spectrum to that of a late-type star are two remarkable properties of LRNe (Pastorello et al. 2019). The photometric information in particular can be used to study how the spectral energy distribution (SED), the effective temperature, and the photospheric radius evolve with time.

To this aim, the SED is computed for a few representative epochs, and the observed data are fitted by a single black-body function. The early-time (near the blue maximum) SEDs do not contain $u, i, z$-band and NIR observations, while $u$-band data are not available from about two months after peak. Finally, the fluxes in the blue optical bands are not available at very late phases, because the object was below the detection thresholds in those filters. The resulting black-body fits are shown in Fig. 4 (left panel). Until about three months after maximum, 

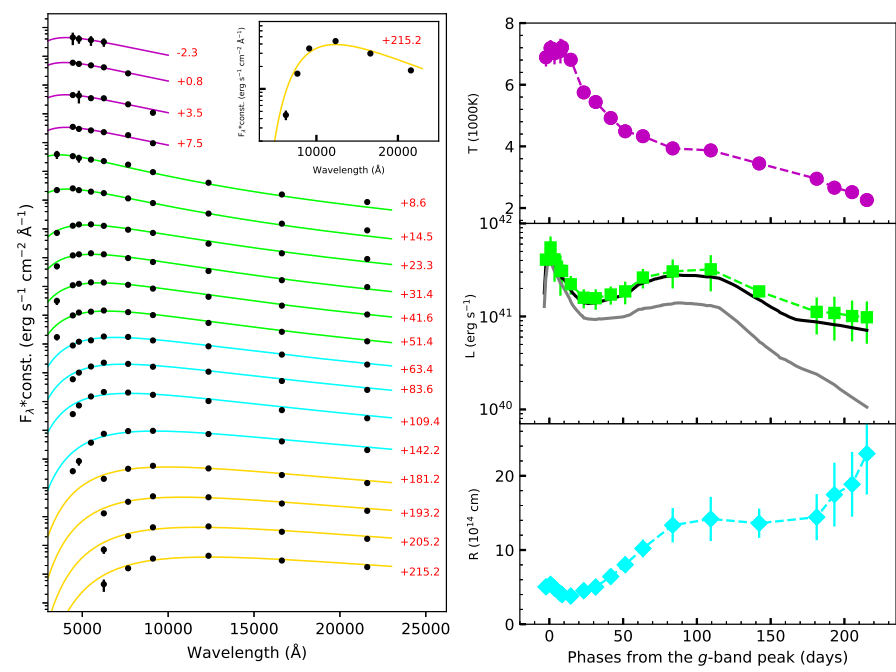

Fig. 4. Left: evolution of the SED at some selected epochs spanning the entire evolution of AT 2017jfs. Top-right: evolution of the effective temperature. Middle-right: bolometric light curve of AT 2017jfs (green squares and dashed line), compared with the uvoir (black solid line) and opt (gray solid line) pseudo-bolometric curves (see text). Bottom-right: evolution of the photospheric radius of AT 2017jfs.

observations are well modeled by black-body fits, although from 3-4 weeks the line-blanketed $u$-band sits below the adopted models. After the red peak, a single black body is not sufficient to accurately represent the observed SEDs in the blue region (see the inset in the left panel of Fig. 4). This happens when the NIR light curves of AT 2017jfs start a new rise before the object is in heliacal conjunction. This is possibly due to the contribution of a second black-body component peaking at longer wavelengths that cannot be properly fitted because of the inadequate wavelength coverage of our observed SED, in particular towards the mid- and far-infrared domains. The nature of this putative cold component is unclear. It is possibly due to an IR echo from distant pre-existing dust or, more likely, to the condensation of newly formed dust, as suggested by the early appearance of molecular bands in the spectra and the strong blue-shift of the late $\mathrm{H} \alpha$ emission (Sect. 4).

The evolution of the effective temperature is shown in the top-right panel of Fig. 4. The temperature remains roughly constant at about $7000 \mathrm{~K}$ during the blue peak. Soon after maximum, the temperature declines very rapidly, reaching $\sim 4500 \mathrm{~K}$ at $50 \mathrm{~d}$. Later on, the temperature fades more slowly, down to about $2300 \mathrm{~K}$ at $215 \mathrm{~d}$, although this value is uncertain, as it was inferred from a poor, single black-body fit (see inset in Fig. 4, left panel).

The temporal evolution of the bolometric luminosity of AT 2017jfs, inferred by integrating the black-body fluxes over the entire wavelength range, is shown in Fig. 4 (mid-right panel), and is compared with the pseudo-bolometric curves obtained by accounting for the contribution of the optical plus NIR bands (uvoir), and the optical bands (opt) only. For the first peak we obtain a bolometric luminosity $L_{\text {bol }} \sim 5.5 \times 10^{41} \mathrm{erg} \mathrm{s}^{-1}$, which is comparable to those of other intermediate-luminosity optical transients (Berger et al. 2009; Soker \& Kashi 2012) or faint core-collapse SNe (Pastorello et al. 2004; Spiro et al. 2014). After the post-peak decline (with a minimum of $L_{\text {bol }} \sim 1.5 \times$ $10^{41} \mathrm{erg} \mathrm{s}^{-1}$ ), the bolometric light curve rises to the second peak with $L_{\text {bol }} \sim 3.2 \times 10^{41} \mathrm{erg} \mathrm{s}^{-1}$, and then declines again. After $\sim 150 \mathrm{~d}$, in coincidence with the late NIR brightening, the bolometric light curve flattens to $L_{\mathrm{bol}} \sim 10^{41} \mathrm{erg} \mathrm{s}^{-1}$. We note that the bolometric luminosity of the blue peak in AT 2017jfs is twice that of the red peak. This is a major difference with NGC 4490-2011OT1, where the red peak was twice as luminous as the early blue peak (see Pastorello et al. 2019, their Fig. 11). This discrepancy is likely a consequence of the large UV contribution during the blue peak that was not accounted for in the pseudo-bolometric light curve of NGC 4490-2011OT1.

Using the Stefan-Boltzmann law, with the luminosities and temperatures estimated above, we infer the evolution of the radius at the photosphere for AT 2017jfs (Fig. 4, bottom-right panel). The radius $R$ at blue peak slightly exceeds $5 \times 10^{14} \mathrm{~cm}$ $\left(R \approx 7700 R_{\odot}\right)$. After a modest decline, the radius rapidly increases reaching $\sim 19000 R_{\odot}$ at about $80 \mathrm{~d}$, and then remains roughly constant until $\sim 6$ months. During the last month of the monitoring campaign of AT 2017jfs, we observe a further fast increase in the photospheric radius, which exceeds $33000 R_{\odot}$ at $215 \mathrm{~d}$. This rise in the photospheric radius and the dramatic decline of the effective temperature at very late phases favor the formation of new dust, like in RN V838 Mon (Bond 2003). This is also consistent with the blueshift of the $\mathrm{H} \alpha$ emission shown in Fig. 2 (left).

\section{Discussion and conclusions}

Pastorello et al. (2019) presented optical data for a wide sample of extra-galactic LRNe, all of them showing double-peaked light curves with maximum absolute magnitudes $M_{V}$ in the range -12.5 to $-15 \mathrm{mag}$. They discuss the observational similarity of LRNe with fainter $\left(M_{V}>-10 \mathrm{mag}\right) \mathrm{RNe}$ discovered in the Milky Way, and agree with Kochanek et al. (2014) and Smith et al. (2016) that all these transients are explained in a similar binary-interaction framework. Most likely, they result from stellar merging events that occurred after the ejection of the common envelope. Lipunov et al. (2017) and MacLeod et al. (2017) discuss the structured light curves of LRNe. A plausible scenario for the double-peak light curve of AT 2017jfs invokes an initial mass outflow as a consequence of the merging event, followed by a later interaction with the common envelope. This would produce the first luminosity peak and the spectra resembling those of type-IIn SNe. During the second peak, the photospheric radius $\left(\sim 2 \times 10^{4} R_{\odot}\right)$ is likely coincident with that of the ejected common envelope. With the temperature decline, the $\mathrm{H}$ recombines, and the released radiation determines the broad red maximum. According to Metzger \& Pejcha (2017), the doublepeak light curve of LRNe is explained with a modest mass ejection following the coalescence, with the early peak being due to the release of thermal energy from the fast ejecta in free expansion along the polar axes. The late red peak would result from shock-powered emission in the collision between the fast shell and pre-existing material in the equatorial plane. This would also generate a cool dense shell, which is an ideal site for late dust formation, as likely observed in AT 2017jfs. Barsukova et al. (2014) provided a somewhat different interpretation. The rapid coalescence generates a violent forward shock which leads the photospheric temperature to largely increase, producing the blue light curve peak. This phase is followed by the fast adiabatic expansion of the envelope with thermal energy carried out with some delay to the outer layers producing the broad red maximum.

Kochanek et al. (2014) proposed that the wide range of peak luminosities observed in RN/LRN events (over 4 orders of magnitudes in luminosity) is tightly connected with the total mass of the binary system, with faint $\mathrm{RNe}$ having progenitor systems of the order of $1 M_{\odot}$ and intermediate-luminosity events like V838 Mon of $\lesssim 10 M_{\odot}$. Luminous transients such as AT 2017jfs, 
SNhunt248 (Mauerhan et al. 2015; Kankare et al. 2015), and NGC 4490-2011OT1 (Smith et al. 2016; Pastorello et al. 2019) likely arise from more massive binaries (up to $50-60 M_{\odot}$, Mauerhan et al. 2018). While for AT 2017jfs we do not have any direct information on the progenitor system and the preoutburst light-curve evolution, its luminous light curve would favor a massive binary as precursor of AT 2017jfs.

A possible correlation between outflow velocities and lightcurve peak luminosities for merger candidates is presented in Mauerhan et al. (2018), but includes intermediate-luminosity red transients similar to SN 2008S and M85-OT whose nature is debated (e.g., Botticella et al. 2009; Kasliwal et al. 2011; Kulkarni et al. 2007; Pastorello et al. 2007). Since for AT 2017jfs we measure an expansion velocity $v_{F W H M} \approx 700 \mathrm{~km} \mathrm{~s}^{-1}$ and $\log \left(L_{\text {peak }} / L_{\odot}\right) \approx 7.2$, the object is positioned very close to NGC 4490-2011OT1 in their Fig. 12, hence supporting the parameters trend discussed in Mauerhan et al. (2018).

While $\mathrm{RNe}$ from relatively low-mass stars are expected to be quite common, luminous events are extremely rare (Kochanek et al. 2014). In particular, following Kochanek et al. (2014, their Fig. 3), AT 2017jfs-like events would occur at a rate of $<10^{-4} \mathrm{yr}^{-1}$ within $1 \mathrm{Mpc}$. Therefore, within a volume of $40 \mathrm{Mpc}$ in radius, we should find about three events per year, which is roughly consistent with observations. In fact, while we observed at least four RNe with $M_{V} \gtrsim-10$ mag in the Milky Way in the past two decades (V4332 Sgr, V838 Mon, V1309 Sco and OGLE-2002-BLG-360), LRNe brighter than $M_{V}<-10 \mathrm{mag}$ were never discovered in our Galaxy, with only less that ten objects observed within $40 \mathrm{Mpc}$ in the past few years.

Due to the limited number of objects discovered so far and incomplete data sets, $\mathrm{RNe} / \mathrm{LRNe}$ are still not fully understood. Well-sampled, multi-band light curves extending to longer wavelengths and high-S/N spectra with good resolution are essential tools for improving their characterization. Discovering new LRNe at larger distances and RNe outside the Local Group is crucial for understanding the physics of these objects, and for providing reliable intrinsic rates. These are key objectives of the Large Synoptic Survey Telescope (LSST Science Collaboration 2009) and other future-generation surveys.

Acknowledgements. We thank Rubina Kotak for useful suggestions. YZC is supported by the China Scholarship Council (No. 201606040170). MF is supported by a Royal Society - Science Foundation Ireland University Research Fellowship. NER acknowledges support from the Spanish MICINN grant ESP2017-82674-R and FEDER funds. S.Bose, PC and SD acknowledge Project 11573003 supported by NSFC. This research uses data obtained through the Telescope Access Program (TAP), which has been funded by the National Astronomical Observatories of China, the Chinese Academy of Sciences, and the Special Fund for Astronomy from the Ministry of Finance. S.Benetti is partially supported by PRIN-INAF 2017 "Toward the SKA and CTA era: discovery, localization, and physics of transient sources." (PI: M. Giroletti). KM acknowledges support from STFC (ST/M005348/1) and from H2020 through an ERC Starting Grant (758638). AF acknowledges the support of an ESO Studentship. AMT acknowledges the support from the Program of development of M.V. Lomonosov Moscow State University (Leading Scientific School "Physics of stars, relativistic objects and galaxies". CT, AdUP, DAK and LI acknowledge support from the Spanish research project AYA2017-89384-P, and from the "Center of Excellence Severo Ochoa" award for the IAA (SEV-2017-0709). CT and AdUP acknowledge support from funding associated to Ramón y Cajal fellowships (RyC-2012-09984 and RyC2012-09975). DAK and LI acknowledge support from funding associated to Juan de la Cierva Incorporación fellowships (IJCI-2015-26153 and IJCI-201630940). The Pan-STARRS1 Surveys (PS1) have been made possible through contributions of the Institute for Astronomy, the University of Hawaii, the Pan-STARRS Project Office, the Max-Planck Society and its participating institutes, the Max Planck Institute for Astronomy, Heidelberg and the Max Planck Institute for Extraterrestrial Physics, Garching, The Johns Hopkins University, Durham University, the University of Edinburgh, Queen's University Belfast, the Harvard-Smithsonian Center for Astrophysics, the Las Cumbres
Observatory Global Telescope Network Incorporated, the National Central University of Taiwan, STScI, NASA under Grant No. NNX08AR22G issued through the Planetary Science Division of the NASA Science Mission Directorate, the US NSF under Grant No. AST-1238877, the University of Maryland, and Eotvos Lorand University (ELTE). Operation of the Pan-STARRS1 telescope is supported by NASA under Grant No. NNX12AR65G and Grant No. NNX14AM74G issued through the NEO Observation Program. This paper is also based upon work supported by AURA through the National Science Foundation under AURA Cooperative Agreement AST 0132798 as amended. ATLAS observations were supported by NASA grant NN12AR55G. NUTS is supported in part by the Instrument Center for Danish Astrophysics (IDA). This work is based on observations collected at the European Organisation for Astronomical Research in the Southern Hemisphere under ESO programmes 199.D-0143(G,I,K,L). This work makes use of observations from the LCOGT network. It is also based on observations made with the $2.2 \mathrm{~m}$ MPG telescope at the La Silla Observatory, the Nordic Optical Telescope (NOT), operated on the island of La Palma jointly by Denmark, Finland, Iceland, Norway, and Sweden, in the Spanish Observatorio del Roque de los Muchachos of the Instituto de Astrofísica de Canarias; the $1.82 \mathrm{~m}$ Copernico Telescope of INAF-Asiago Observatory; the Gran Telescopio Canarias (GTC), installed in the Spanish Observatorio del Roque de los Muchachos of the Instituto de Astrofísica de Canarias, in the Island of La Palma; the Liverpool Telescope operated on the island of La Palma by Liverpool John Moores University at the Spanish Observatorio del Roque de los Muchachos of the Instituto de Astrofísica de Canarias with financial support from the UK Science and Technology Facilities Council; the $6 \mathrm{~m}$ Big Telescope Alt-azimuth and the Zeiss-1000 Telescope of the Special Astrophysical Observatory, Russian Academy of Sciences. We thank Las Cumbres Observatory and its staff for their continued support of ASAS-SN. ASAS-SN is supported by the Gordon and Betty Moore Foundation through grant GBMF5490 to the Ohio State University and NSF grant AST-1515927. Development of ASAS-SN has been supported by NSF grant AST-0908816, the Mt. Cuba Astronomical Foundation, the Center for Cosmology and AstroParticle Physics at the Ohio State University, the Chinese Academy of Sciences South America Center for Astronomy (CAS-SACA), the Villum Foundation, and George Skestos.

\section{References}

Banerjee, D. P. K., Nuth, III., J. A., Misselt, K. A., et al. 2015, ApJ, 814, 109

Barsukova, E. A., Goranskij, V. P., Valeev, A. F., \& Zharova, A. V. 2014, Astrophys. Bull., 69, 67

Berger, E., Soderberg, A. M., Chevalier, R. A., et al. 2009, ApJ, 699, 1850

Blagorodnova, N., Kotak, R., Polshaw, J., et al. 2017, ApJ, 834, 107

Bond, H. E. 2003, Nature, 422, 405

Botticella, M. T., Pastorello, A., Smartt, S. J., et al. 2009, MNRAS, 398, 1041

Bufano, F., Berton, M., Vogl, C. 2018, ATel, 11135

Cai, Y.-Z., Pastorello, A., Fraser, M., et al. 2018, MNRAS, 480, 3424

Cappellaro, E. 2014, SNOoPy: A Package for SN Photometry, http:// sngroup.oapd.inaf.it/snoopy.html

Crause, L. A., Lawson, W. A., Kilkenny, D., et al. 2003, MNRAS, 341, 785

Delgado, A., et al. 2007, TNS Report n. 15585

Evans, A., Geballe, T. R., Rushton, M. T., et al. 2003, MNRAS, 343, 1054

Exter, K. M., Cox, N. L. J., Swinyard, B. M., et al. 2016, A\&A, 596, A96

Goranskij, V. P., Shugarov, S Yu, Barsukova, E. A., et al. 2002, Astron. Lett., 28, 691

Greiner, J., Bornemann, W., Clemens, C., et al. 2008, PASP, 120, 405

Jordi, K., Greber, E. K., \& Ammon, K. 2005, A\&A, 460, 339

Jordi, K., Gebran, M., Carrasco, J. M., et al. 2010, A\&A, 523, A48

Kankare, E., Kotak, R., Pastorello, A., et al. 2015, A\&A, 581, L4

Kasliwal, M., Kulkarni, S. R., Arcavi, I., et al. 2011, ApJ, 730, 134

Kimeswenger, S., Lederle, C.. Schmeja, S.. \& Armsdorfer, B. 2002, MNRAS, 336, L43

Kirkpatrick, J. D., Henry, T. J., \& McCarthy, Jr., D. W. 1991, ApJS, 77, 41

Kochanek, C. S., Adams, S. M., \& Belczynski, K. 2014, MNRAS, 443, 1319

Kulkarni, S. R., Ofek, E. O., Rau, A., et al. 2007, Nature, 447, 458

Koliopanos, F., Ciambur, B., Graham, A. W., et al. 2017, A\&A, 601, A20

Lipunov, V. M., Blinnikov, S., Gorbovskoy, E., et al. 2017, MNRAS, 470, 2339

LSST Science Collaboration 2009, ArXiv e-prints [arXiv:0912.0201]

MacLeod, M., Macias, P., Ramirez-Ruiz, E., et al. 2017, ApJ, 835, 282

MacLeod, M., Ostriker, E. C., \& Stone, J. M. 2018, ApJ, 868, 136

Martini, P., Wagner, R., Tomaney, A., et al. 1999, AJ, 119, 1034

Mason, E., Diaz, M., Williams, R. E., et al. 2010, A\&A, 516, A108

Mauerhan, J. C., Van Dyk, S. D., Graham, M. L., et al. 2015, MNRAS, 447, 1922

Mauerhan, J. C., Van Dyk, S. D., Johansson, J., et al. 2018, MNRAS, 473, 3765 
Metzger, B. D., \& Pejcha, O. 2017, MNRAS, 471, 3200

Munari, U., Henden, A., Kiyota, S., et al. 2002, A\&A, 389, L51

Munari, U., Navasardyan, H., \& Villanova, S. 2007, ASPC, 363, 13

Pastorello, A., Benetti, S., Brown, P. J., et al. 2004, MNRAS, 347, 74

Pastorello, A., Della Valle, M., Smartt, S. J., et al. 2007, Nature, 449, 1

Pastorello, A., Reguitti, A., Morales-Garoffolo, A., et al. 2019, A\&A, submitted

Pejcha, O., Metzger, B. D., \& Tomida, K. 2016, MNRAS, 461, 2527

Pejcha, O., Metzger, B. D., Tyles, J. G., \& Tomida, K. 2017, ApJ, 850, 59

Schlafly, E. F., \& Finkbeiner, D. P. 2011, ApJ, 737, 103

Shappee, B. J., Prieto, J. L., Grupe, D., et al. 2014, ApJ, 788, 48

Smartt, S. J., Valenti, S., Fraser, M., et al. 2015, A\&A, 579, A40

Smith, N., Andrews, J. E., Van Dyk, S. D., et al. 2016, MNRAS, 458, 950

Soker, N., \& Kashi, A. 2012, ApJ, 746, 100

Spiro, S., Pastorello, A., Pumo, M. L., et al. 2014, MNRAS, 439, 2873

Tonry, J. L., Denneau, L., Heinze, A. N., et al. 2018, PASP, 130, 064505

Tylenda, R., Hajduk, M., Kamiński, T., et al. 2011, A\&A, 528, A114

Tylenda, R., Hajduk, M., Kamiński, T., et al. 2013, A\&A, 555, A16

Valenti, J. A., Piskunov, N., \& Johns-Krull, C. M. 1998, ApJ, 498, 851

1 INAF - Osservatorio Astronomico di Padova, Vicolo dell'Osservatorio 5, 35122 Padova, Italy e-mail: andrea.pastorello@inaf.it

2 Max-Planck-Institut für Extraterrestrische Physik, Giessenbachstraße 1, 85748 Garching bei München, Germany

3 Dipartimento di Fisica e Astronomia, Università di Padova, Vicolo dell'Osservatorio 3, 35122 Padova, Italy

4 Department of Applied Physics, University of Cádiz, Campus of Puerto Real, 11510 Cádiz, Spain

5 Instituto de Astrofísica de Andalucía (IAA-CSIC), Glorieta de la Astronomía s/n, 18008 Granada, Spain

6 INAF - Osservatorio Astronomico di Trieste, Via G.B. Tiepolo 11, 34143 Trieste, Italy

7 Special Astrophysical Observatory, Russian Academy of Sciences, Nizhnij Arkhyz, Karachai-Cherkesia 369167, Russia

8 Finnish Centre for Astronomy with ESO (FINCA), University of Turku, Quantum, Vesilinnantie 5, 20014 Turku, Finland

9 Aalto University Metsähovi Radio Observatory, Metsähovintie 114, 02540 Kylmälä, Finland

10 Kavli Institute for Astronomy and Astrophysics, Peking University, Yi He Yuan Road 5, Hai Dian District, Beijing 100871, PR China

11 INAF - Osservatorio Astrofisico di Catania, Via Santa Sofia 78, 95123 Catania, Italy

12 School of Physics, O'Brien Centre for Science North, University College Dublin, Belfield Dublin 4, Ireland

13 SRON, Netherlands Institute for Space Research, Sorbonnelaan 2, 3584 CA Utrecht, The Netherlands
14 Department of Astrophysics/IMAPP, Radboud University, PO Box 9010, 6500 GL Nijmegen, The Netherlands

15 Cerro Tololo Inter-American Observatory, National Optical Astronomy Observatory, Casilla 603, La Serena, Chile

16 Nordic Optical Telescope, Apartado 474, 38700 Santa Cruz de La Palma, Santa Cruz de Tenerife, Spain

17 Institute of Space Sciences (ICE, CSIC), Campus UAB, Camí de Can Magrans s/n, 08193 Cerdanyola del Vallès (Barcelona), Spain

18 Institut d'Estudis Espacials de Catalunya (IEEC), c/Gran Capità 24, Edif. Nexus 201, 08034 Barcelona, Spain

19 European Southern Observatory, Karl-Schwarzschild-Straße 2, 85748 Garching bei München, Germany

20 Max-Planck-Institut für Astrophysik, Karl-Schwarzschild-Straße 1, 85748 Garching bei München, Germany

21 Physik-Department, Technische Universität München, JamesFranck-Straße 1, 85748 Garching bei München, Germany

22 Gran Telescopio Canarias (GRANTECAN), Cuesta de San José s/n, 38712 Breña Baja, La Palma, Spain

23 Instituto de Astrofísica de Canarias, Vía Láctea s/n, $38200 \mathrm{La}$ Laguna, Tenerife, Spain

24 Sternberg Astronomical Institute, Lomonosov Moscow University, Universitetsky Ave. 13, 119992 Moscow, Russia

25 Tuorla Observatory, Department of Physics and Astronomy, University of Turku, 20014 Turku, Finland

26 Istituto di Astrofisica e Planetologia Spaziali (INAF), Via del Fosso del Cavaliere 100, 00133 Roma, Italy

27 Departamento de Ciencias Físicas, Universidad Andrés Bello, Santiago, Chile

28 Nordita, KTH Royal Institute of Technology and Stockholm University, 10691 Stockholm, Sweden

29 Department of Astronomy, AlbaNova University Center, Stockholm University, 10691 Stockholm, Sweden

30 The Oskar Klein Centre, Department of Physics, Stockholm University, AlbaNova, 10691 Stockholm, Sweden

31 Astrophysics Research Centre, School of Mathematics and Physics, Queen's University Belfast, Belfast BT7 1NN, UK

32 Kazan Federal University, Kremlevskaya 18, 420008 Kazan, Russia

33 Institute of Astronomy, University of Cambridge, Madingley Road, Cambridge CB3 0HA, UK

34 Dark Cosmology Centre, Niels Bohr Institute, University of Copenhagen, Juliane Maries Vej 30, 2100 Copenhagen, Denmark

35 School of Physics \& Astronomy, Cardiff University, Queens Buildings, The Parade, Cardiff CF24 3AA, UK

36 School of Physics, Trinity College Dublin, Dublin 2, Ireland

37 LSST, 950 North Cherry Avenue, Tucson AZ 85719, USA

38 The Oskar Klein Centre, Department of Astronomy, Stockholm University, AlbaNova, 10691 Stockholm, Sweden 


\section{Appendix A: Additional data}

Table A.2. General information on the spectra of AT 2017jfs.

\begin{tabular}{lllllll}
\hline \hline Date & MJD & Phase (days) & Instrumental configuration & Exptime $(\mathrm{s})$ & Res $(\AA)$ & Range $(\AA)$ \\
\hline 2018 Jan 6 & 58124.31 & +9.5 & NTT+EFOSC2+gm13 & 600 & 18 & $3650-9200$ \\
2018 Jan 10 & 58128.26 & +13.5 & NOT+ALFOSC+gm4 & 3600 & 18 & $3600-9650$ \\
2018 Jan 17 & 58135.33 & +20.5 & NTT+EFOSC2+gm11 & $2 \times 3600$ & 14 & $3350-7450$ \\
2018 Jan 20 & 58138.16 & +23.4 & NOT+ALFOSC+gm4 & 3600 & 14 & $3500-9700$ \\
2018 Jan 24 & 58142.41 & +27.6 & P200+DBSP+gt316/7500 & $2 \times 0$ & 5.5 & $5400-9500$ \\
2018 Feb 13 & 58162.22 & +47.4 & NTT+EFOSC2+gm11 & G & 14 & $3350-7450$ \\
2018 Feb 23 & 58172.10 & +57.3 & GTC+OSIRIS+R1000B+R1000R & $1800+1800$ & $7.0+7.8$ & $3850-9250$ \\
2018 Mar 20 & 58197.10 & +82.3 & GTC+OSIRIS+R1000B+R1000R & $1800+1800$ & $7.0+7.8$ & $3650-9350$ \\
2018 Apr 22 & 58230.94 & +116.1 & BTA+SCORPIO+VPHG550G & $2 \times 1200$ & 13 & $3650-7900$ \\
2018 Apr 29 & 58237.05 & +122.3 & GTC+OSIRIS+R1000B+R1000R & $2 \times 900+2 \times 900$ & $7.0+7.8$ & $3650-9200$ \\
2018 May 23 & 58261.06 & +146.3 & GTC+OSIRIS+R1000B+R1000R & $2 \times 1600+1600$ & $7.0+7.8$ & $3650-9250$ \\
2018 Jun 1 & 58270.98 & +156.2 & GTC+OSIRIS+R1000R & $2 \times 1800$ & 7.8 & $5100-9350$ \\
2018 Jun 2 & 58271.97 & +157.2 & GTC+OSIRIS+R1000R & $2 \times 1800$ & 7.8 & $5100-9350$ \\
2018 Jun 16 & 58285.93 & +171.1 & GTC+OSIRIS+R1000R & $4 \times 1500$ & 7.8 & $5100-9250$ \\
\hline
\end{tabular}

Notes. The phases are from the $g$-band maximum. NTT $=3.58 \mathrm{~m}$ New Technology Telescope (ESO-La Silla, Chile); NOT $=2.56 \mathrm{~m}$ Nordic Optical Telescope (La Palma, Canary Islands, Spain); P200 = 5.1 m (200-inch) Hale Telescope (Mt. Palomar, California, USA); GTC = 10.4 m Gran Telescopio Canarias (La Palma, Canary Islands, Spain); BTA $=6.05 \mathrm{~m}$ Bolshoi Teleskop Alt-azimutalnyi (Special Astrophysical Observatory, Karachay-Cherkessian Republic, Russia). 